\title{
Down syndrome critical region 1 enhances the proteolytic cleavage of calcineurin
}

\author{
Ji-Eun Lee ${ }^{1 *}$, Hyonchol Jang ${ }^{1 *}$, \\ Eun-Jung $\mathrm{Cho}^{2}$ and Hong-Duk Youn ${ }^{1,3}$
}

${ }^{1}$ National Research Laboratory for Metabolic Checkpoint

Department of Biomedical Sciences and

Biochemistry and Molecular Biology

Cancer Research Institute

Seoul National University College of Medicine

Seoul 110-799, Korea

${ }^{2}$ National Research Laboratory for Chromatin Dynamics

College of Pharmacy

Sungkyunkwan University

Suwon 440-746, Korea

${ }^{3}$ Corresponding author: Tel, 82-2-740-8250;

Fax, 82-2-3668-7622; E-mail, hdyoun @ snu.ac.kr

*These authors contributed equally to this work.

DOI 10.3858/emm.2009.41.7.052

Accepted 9 February 2009

Abbreviations: AD, Alzheimer's disease; AKAP79, A-kinase anchoring protein-79, CHP, CNB homologous protein; DS, Down syndrome; DSCR1, down syndrome critical region 1; HEK, human embryonic kidney

\begin{abstract}
Down syndrome critical region 1 (DSCR1), an oxidative stress-response gene, interacts with calcineurin and represses its phosphatase activity. Recently it was shown that hydrogen peroxide inactivates calcineurin by proteolytic cleavage. Based on these facts, we investigated whether oxidative stress affects DSCR1mediated inactivation of calcineurin. We determined that overexpression of DSCR1 leads to increased proteolytic cleavage of calcineurin. Convertsely, knockdown of DSCR1 abolished calcineurin cleavage upon treatment with hydrogen peroxide. The PXIIXT motif in the $\mathrm{COOH}$-terminus of DSCR1 is responsible for both binding and cleavage of calcineurin. The knockdown of overexpressed DSCR1 in DS fibroblast cells also abrogated calcineurin proteolysis by hydrogen peroxide. These results suggest that DSCR1 has the ability to inactivate calcineurin by inducing proteolytic cleavage of calcineurin upon oxidative stress.
\end{abstract}

Keywords: calcineurin; Down syndrome; DSCR1 protein, mouse; fibroblasts; hydrogen peroxide; oxidative stress; RCAN1 protein, human

\section{Introduction}

Calcineurin (also known as protein phosphatase 3), a calcium and calmodulin-dependent protein serine/ threonine phosphatase plays a critical role in various biological processes. Calcineurin is involved in the regulation of $T$ cell development, heart valve and myocardial development, and in neuronal memory and learning (Aramburu et al., 2000; Crabtree 2001). Calcineurin is also related with Alzheimer's disease (AD). Calcineurin, whose phosphatase activity is decreased in $A D$, dephosphorylates $\tau$, which is abnormally phosphorylated in AD (Gong et al., 1994).

Down syndrome critical region 1 (DSCR1) belongs to a family of evolutionarily conserved proteins that can directly bind and inhibit calcineurin (Fuentes et al., 2000; Kingsbury and Cunningham, 2000; Ermak et al., 2002). DSCR1 is involved in the regulation of various cellular functions (Harris et al., 2005). It is overexpressed in the brains of Down Syndrome (DS) fetuses and in post-mortem brain samples from AD patients (Fuentes et al., 2000; Ermak et al., 2001). DSCR1 has also been implicated in cardiac valve formation and in inhibition of cardiac hypertrophy (Yang et al., 2000; Lange et al., 2004). DSCR1, induced by VEGF, TNF- $\alpha$, and calcium ionophore, participates in endothelial cell migration and angiogenesis (lizuka et al., 2004; Yao and Duh, 2004).

In addition to DSCR1, other endogenous proteins such as A-kinase anchoring protein-79 (AKAP79), Cabin1, and CNB homologous protein (CHP) also inhibit calcineurin activity (Lin and Barber, 1996; Kashishian et al., 1998; Lai et al., 1998). Unlike these inhibitors, DSCR1 has a unique feature as a dual regulator of calcineurin (Vega et al., 2003). Both overexpression and deletion of DSCR1 impairs calcineurin activity (Kingsbury and Cunningham, 2000; Vega et al., 2003). As far as we know, however, naturally occurring DSCR1-related pathologies are limited to its overexpression in cases like DS and $A D$. Therefore, our major concern is the mechanism of calcineurin inhibition in conditions of DSCR1 overexpression.

To date, many studies have focused on the 
inhibitory mechanism of interaction between calcineurin and DSCR1. For example, a previous study showed that DSCR1 binds to the catalytic domain of the CNA subunit (Rothermel et al., 2000). Although some subtle discrepancies in the binding domain exist among these studies, the C-terminal half of DSCR1 is responsible for this interaction in all studies (Rothermel et al., 2000; Vega et al., 2002; Chan et al., 2005; Aubareda et al., 2006). There is no doubt that this direct interaction is an important mechanism for inhibition. However, this mode of inhibition is usually reversible. In our previous study, we demonstrated that $\mathrm{H}_{2} \mathrm{O}_{2}$ triggers proteolytic cleavage and irreversible inactivation of calcineurin (Lee et al., 2007). Since DSCR1 exhibits greater stability with $\mathrm{H}_{2} \mathrm{O}_{2}$ (Michtalik et al., 2004), we hypothesized that DSCR1 may be involved in the proteolysis of calcineurin in conditions of oxidative stress. To test this hypothesis, we overexpressed or down-regulated DSCR1 and examined the calcineurin protein content in the presence of $\mathrm{H}_{2} \mathrm{O}_{2}$.

Here we show that overexpression of DSCR1 increases the proteolytic cleavage of calcineurin and that knockdown of DSCR1 inhibits cleavage of calcineurin after treatment with $\mathrm{H}_{2} \mathrm{O}_{2}$.

\section{Results}

\section{DSCR1 overexpression leads to accelerated cleavage of calcineurin}

Previously, we showed that $\mathrm{H}_{2} \mathrm{O}_{2}$ triggers proteolytic cleavage and inactivation of calcineurin (Lee et al., 2007). When we transfected SK-N-SH cells with mammalian expression vectors for calcineurin and its endogenous inhibitors, we found that DSCR1 overexpression resulted in proteolytic cleavage of calcineurin, similar to $\mathrm{H}_{2} \mathrm{O}_{2}$ (Figure 1A). However, AKAP79 and Cabin1 did not trigger the calcineurin proteolysis (Figure 1A). To confirm this result, we generated recombinant DSCR1 adenovirus (AdDSCR1) and then infected mouse primary cortical neurons with Ad-DSCR1 or Ad-Mock. DSCR1 overexpression not only led to cleavage of endogenous calcineurin, but also accelerated the cleavage of calcineurin upon exposure to $\mathrm{H}_{2} \mathrm{O}_{2}$ (Figure 1B). In the same way, DSCR1 increased the proteolysis of ectopically overexpressed calcineurin (Figure 1C).
A

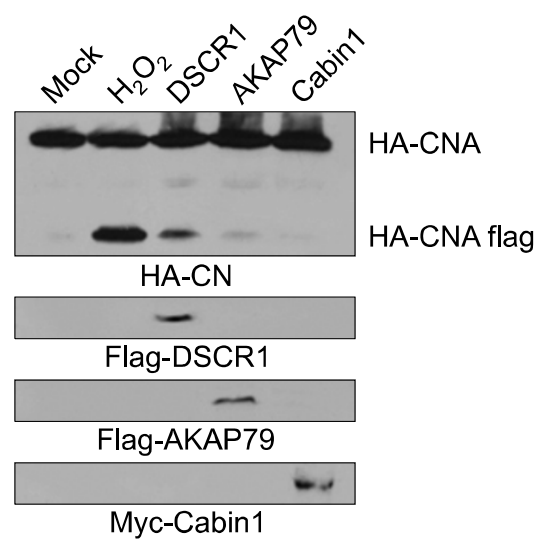

C

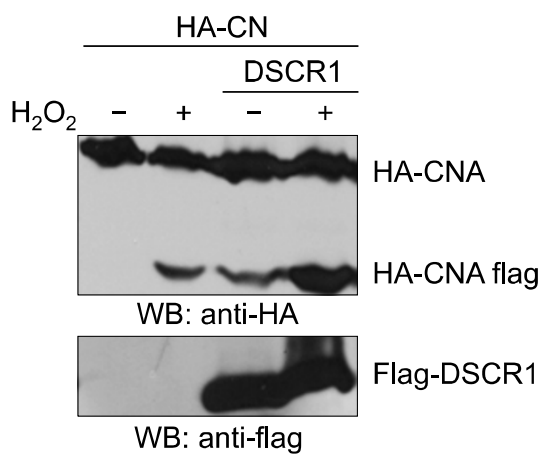

B

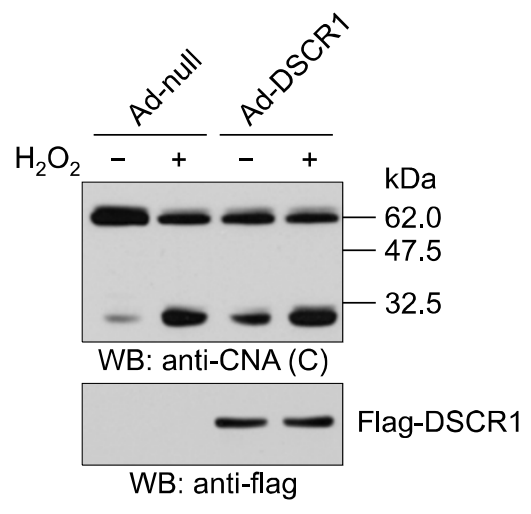

D

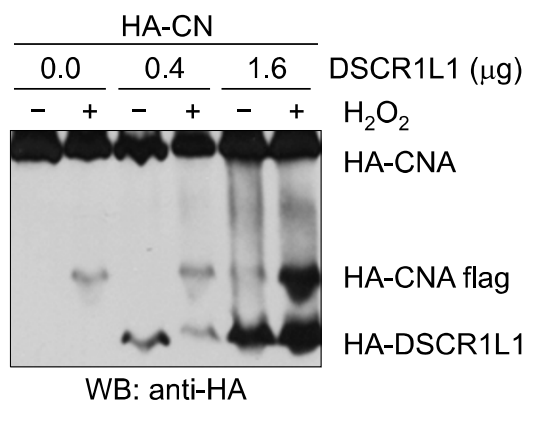

Figure 1. Increase of calcineurin cleavage by DSCR1. (A) Proteolysis of calcineurin by DSCR1 overexpression. SK-N-SH cells expressing endogenous calcineurin inhibitor proteins together with calcineurin were harvested and directly boiled in SDS sample buffer for western blot analysis. (B) Enhancement of endogenous calcineurin cleavage by DSCR1 overexpression. Mouse primary cortical neurons were infected with adenovirus expressing DSCR1 or empty adenovirus vector on the fourth day after plating. Three days later, cells were harvested and cell lysates were prepared by direct boiling in SDS sample buffer. (C) Enhancement of exogenous calcineurin cleavage by DSCR1. pSGHA-calcineurin and pcDNA3-DSCR1FLAG were transfected into HEK293 cells. After $24 \mathrm{~h}$, cells were harvested and directly boiled in SDS sample buffer. (D) Increased proteolysis of calcineurin by DSCR1L1 (ZAKI-4) overexpression. Increasing concentrations of pSG-HA-DSCR1L1 with pSG-HA-calcineurin were transfected into HEK293 cells. After 24 h, cells were harvested and directly boiled in SDS sample buffer. 
To determine whether another member of DSCR family, DSCR1L1 (ZAKI-4), was also able to accelerate $\mathrm{CN}$ cleavage, we transfected SK-N-SH cells with increasing amounts of DSCR1L1 expression plasmid in the presence of a calcineurin expression vector. Increasing amounts of DSCR1L1 expression also increased the level of cleaved $\mathrm{CN}$ fragment (Figure 1D).

\section{DSCR1 knockdown inhibits calcineurin cleavage upon $\mathrm{H}_{2} \mathrm{O}_{2}$ exposure}

To assess the effects of DSCR1 knockdown on calcineurin proteolysis, siRNA was used to reduce DSCR1 levels in the human neuroblastoma cell line SK-N-SH. siRNA that targets GFP sequence was used as a control for nonspecific effects of siRNA transfection. Transient transfection of SK-N-SH cells with the DSCR1 siRNA oligonucleotide reduced DSCR1 mRNA and protein levels (Figure 2, lane 3 and 4). In control GFP knockdown cells, calcineurin was cleaved upon exposure to $\mathrm{H}_{2} \mathrm{O}_{2}$ (Figure 2, lane 2). In DSCR1 knockdown cells, in contrast,

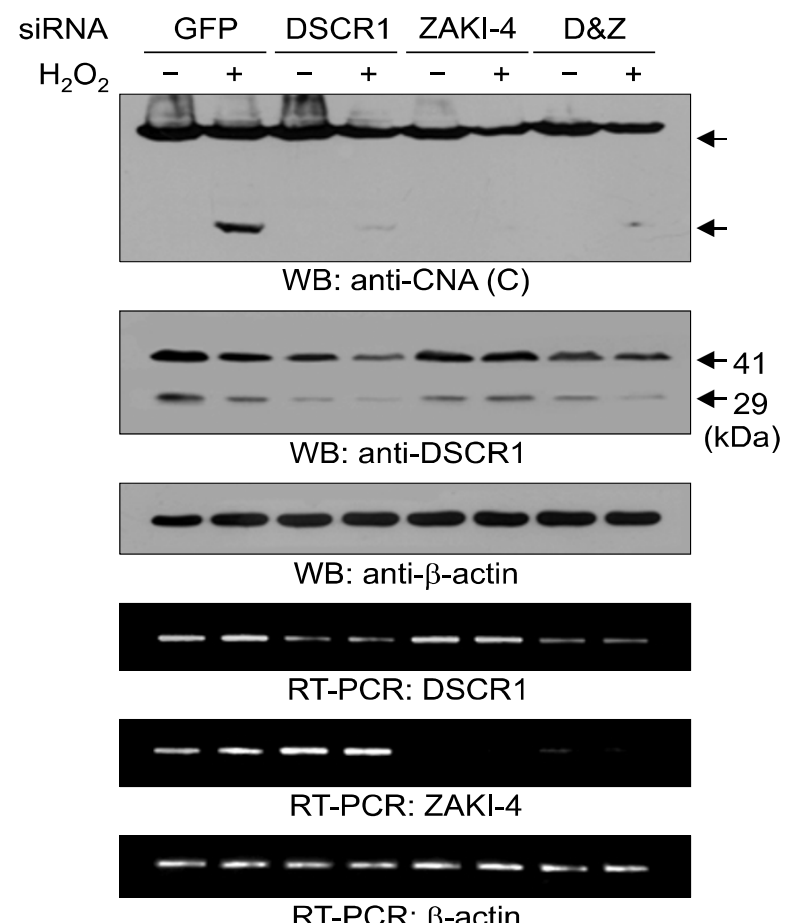

RT-PCR: $\beta$-actin

Figure 2. Abolishment of calcineurin cleavage upon $\mathrm{H}_{2} \mathrm{O}_{2}$ exposure in DSCR1 and DSCR1L1 (ZAKI-4) knockdown cells. siRNA oligonucleotides targeting GFP, DSCR1, DSCR1L1 (ZAKI-4), or DSCR1/ZAKI-4 were transiently transfected into SK-N-SH cells; $48 \mathrm{~h}$ after transfection, cells were treated with $\mathrm{H}_{2} \mathrm{O}_{2}(0.25 \mathrm{mM}, 30 \mathrm{~min})$. Cell lysates were then subjected to western blot analysis. Specific knockdown of target genes was determined by western blot and RT-PCR with gene-specific primers. calcineurin was not cleaved even after $\mathrm{H}_{2} \mathrm{O}_{2}$ treatment (Figure 2, lane 3 and 4), indicating that DSCR1 plays a critical role in $\mathrm{H}_{2} \mathrm{O}_{2}$-mediated proteolysis of calcineurin. To determine whether ZAKI-4 has the same effect on $\mathrm{CN}$ cleavage, we also used ZAKI-4 siRNA. Likewise, CN cleavage was not observed after $\mathrm{H}_{2} \mathrm{O}_{2}$ treatment in ZAKI-4 knockdown cells (Figure 2, lane 6). Because overexpression of either DSCR1 or ZAKI-4 alone could result in proteolytic cleavage of calcineurin, we expected that knockdown of either one alone would not affect the cleavage of calcineurin upon exposure to $\mathrm{H}_{2} \mathrm{O}_{2}$. Contrary to our expectations, knockdown of either DSCR1 or ZAKI-4 alone abolished the calcineurin cleavage following treatment of cells with $\mathrm{H}_{2} \mathrm{O}_{2}$.

\section{Proteolytic cleavage of calcineurin requires interaction between DSCR1 and calcineurin}

Because DSCR1 is known to interact with and regulate calcineurin, we next asked if binding of DSCR1 was involved in calcineurin cleavage. We therefore generated several truncated mutants of DSCR1 (Figure 3A) and assessed their abilities to interact with calcineurin. Deletion of the $\mathrm{N}$-terminus of DSCR1 [DSCR1(C); amino acids 90-197] did not affect calcineurin binding, whereas deletion of the C-terminus of DSCR1 [DSCR1(N); amino acids 1-90] abolished binding to calcineurin (Figure 3B, upper panel). Overexpression of the $\mathrm{C}$-terminus of DSCR1 promoted calcineurin cleavage; however, expression of the $\mathrm{N}$-terminus alone did not result in $\mathrm{CN}$ cleavage (Figure 3B, indicated by an asterisk). The C-terminus of DSCR1, which contains a PXIIXT motif, is reported to interact with calcineurin (Chan et al., 2005; Aubareda et al., 2006). To confirm these results in our system, we performed co-immunoprecipitation assays in HEK293 cells. DSCR1 mutant lacking the FLISPPXSPP motif ( $\triangle$ SPP; amino acids 1-106/121-197) retained calcineurin-binding activity (Figure $3 \mathrm{C}$, left panel, lane $3)$. In contrast, deletion of DSCR1 PXIIXT motif (1-177) diminished the binding to $\mathrm{CN}$ (Figure $3 \mathrm{C}$, left panel, lane 4) and changed the cleavage pattern of calcineurin (Figure 3C, right panel). Truncation of both the FLISPPXSPP motif and the PXIIXT motif abolished the ability to interact with calcineurin. In addition, double truncation also abolished the ability of DSCR1 to mediate calcineurin cleavage (Figure $3 \mathrm{C}$, right panel).

\section{Requirement of DSCR1 for calcineurin proteolysis in DS fibroblast}

To verify the role of DSCR1 in proteolysis of 
calcineurin in physiological conditions, we used skin fibroblasts of a DS patient. Western blot analysis revealed that DSCR1 protein is overexpressed in DS fibroblasts (Figure 4A). We compared the $\mathrm{H}_{2} \mathrm{O}_{2}$-induced cleavage fragment of calcineurin from DS fibroblast lysate with that from normal control fibroblast lysate. There were no significant differences in calcineurin protein levels between DS and control under normal conditions. Interestingly, however, DS fibroblasts treated with $\mathrm{H}_{2} \mathrm{O}_{2}$ exhibited accelerated cleavage of calcineurin compared to control fibroblasts (Figure 4A). In addition, knockdown of overexpressed DSCR1 in DS fibroblast cells resulted in a concomitant reduction in calcineurin cleavage by $\mathrm{H}_{2} \mathrm{O}_{2}$ (Figure 4B). Judging from these data, DSCR1 plays an important role in $\mathrm{H}_{2} \mathrm{O}_{2}$-mediated calcineurin proteolysis under physiological conditions.

\section{Discussion}

DSCR1 interacts with the CNA catalytic subunit and inhibits calcineurin phosphatase activity (Rothermel et al., 2000; Vega et al., 2002; Chan et al., 2005; Aubareda et al., 2006). Here we show that overexpressed DSCR1 can also increase the proteolysis of calcineurin while other inhibitor proteins cannot (Figure 1). This is a completely novel mode of calcineurin inhibition by DSCR1. In many respects, DSCR1 is a unique inhibitor of calcineurin. First, DSCR1 is transcriptionally induced by calcineurin activity and thereby participates in a negative feedback loop (Yang et al., 2000). Second, DSCR1 is a dual regulator of calcineurin as both an inhibitor and an activator. Low concentrations of DSCR1 stimulate calcineurin activity, whereas high concentrations suppress calcineurin activity (Hilioti et al., 2004). Our results suggest another unique feature in that DSCR1 overexpression causes proteolysis of calcineurin.

We previously published that $\mathrm{H}_{2} \mathrm{O}_{2}$ mediates the proteolysis of calcineurin and irreversibly inactivates calcineurin (Lee et al., 2007). $\mathrm{H}_{2} \mathrm{O}_{2}$ has been shown to be a major component of reactive oxygen species (ROS) in cells and the irreversible damages
A

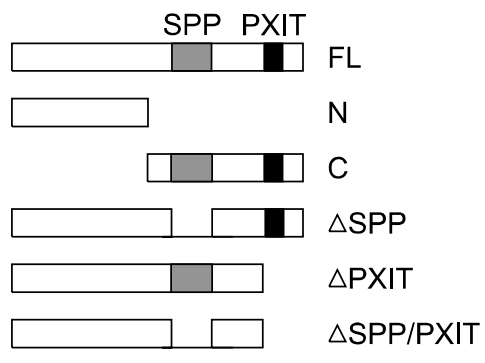

C

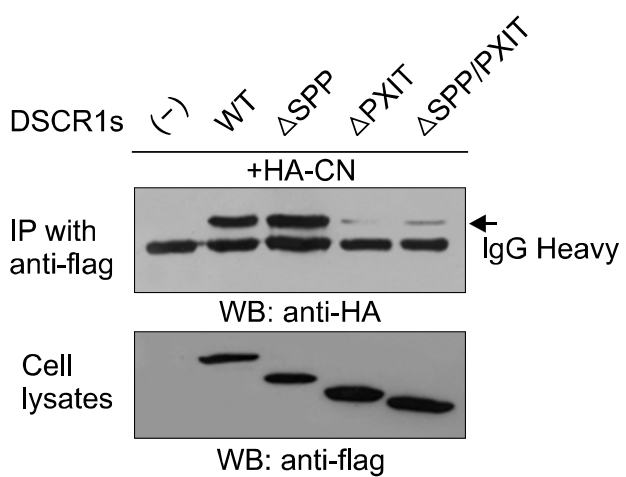

B

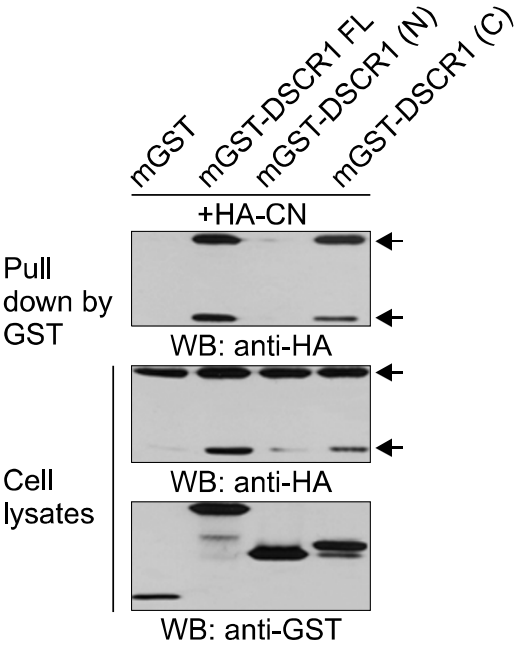

Figure 3. DSCR1 interaction with calcineurin is required for calcineurin proteolysis. (A) A schematic diagram illustrating constructs of fulllength DSCR1 and various truncated mutants of DSCR1. (B) Involvement of the C-terminus of DSCR1 in the interaction with calcineurin and cleavage of calcineurin. Cell lysates expressing mammalian expression vector for GST-DSCR1 truncation mutants together with calcineurin were pulled-down by GST and washed three times with lysis buffer. (C) Involvement of PXIIXT motif in interaction with calcineurin. 
A

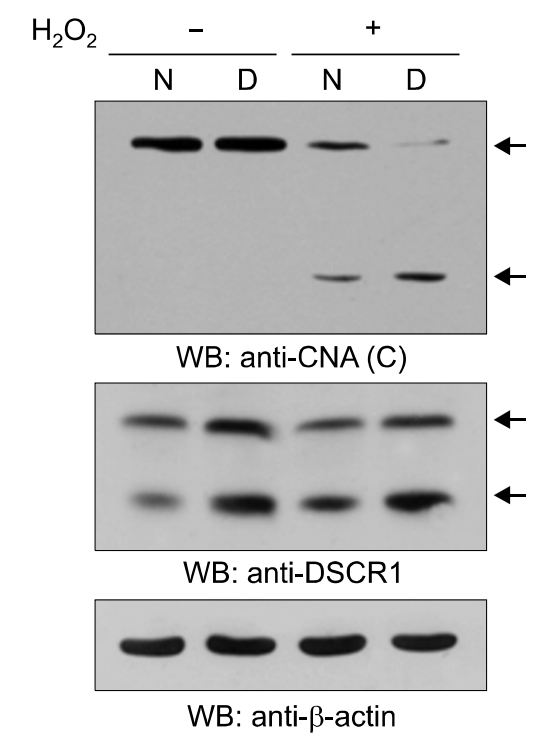

B

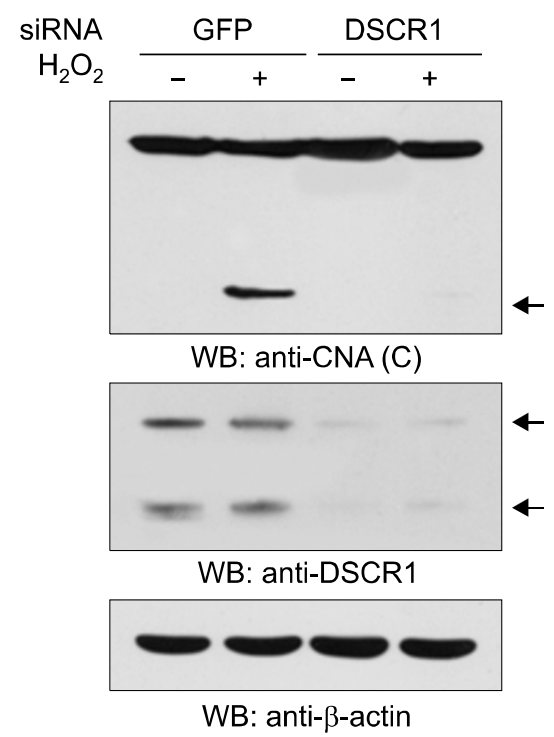

Figure 4. DSCR1 is required for calcineurin proteolysis in Down syndrome fibroblasts. (A) Acceleration of calcineurin proteolysis upon $\mathrm{H}_{2} \mathrm{O}_{2}$ exposure in skin fibroblasts of Down syndrome (DS) patient. Skin fibroblast cell lines from a Down syndrome patient (D) and a normal person $(\mathrm{N})$ were obtained from the Aging Cell Repository of the Coriell Cell Repository (CCR). Down syndrome fibroblast was AG08942 (21year-old male patient) and control fibroblast was AG10803 (22-year-old healthy male). (B) Reduction of calcineurin proteolysis after siRNAmediated depletion of DSCR1 in DS fibroblast. AG08942 DS fibroblast cells were transiently transfected with siRNA for DSCR1 or control GFP; $48 \mathrm{~h}$ after transfection, cells were treated with $\mathrm{H}_{2} \mathrm{O}_{2}(0.25 \mathrm{mM}$, $30 \mathrm{~min}$ ) and lysed. of ROS action have been implicated in a variety of disorders and aging process (Rhee, 1999). Several proteomic- and meta-analyses revealed that $\mathrm{H}_{2} \mathrm{O}_{2}$ induces various cellular proteins (Seong et al., 2002; Vandenbroucke et al., 2008). DSCR1 mRNA is induced by $\mathrm{H}_{2} \mathrm{O}_{2}$ (Crawford et al., 1997), and its protein product is overexpressed in $D S$ and $A D$ brains, which show excessive $\mathrm{H}_{2} \mathrm{O}_{2}$ levels (Fuentes et al., 2000; Ermak et al., 2001). Therefore, DSCR1 is probably involved in calcineurin proteolysis in the $\mathrm{H}_{2} \mathrm{O}_{2}$-rich environment. Although overexpressed DSCR 1 causes proteolytic cleavage of calcineurin in vivo, DSCR1 itself does not have proteolytic activity. When DSCR1 or DSCR1L1 (ZAKI-4) expression was down-regulated using RNA interference, however, $\mathrm{H}_{2} \mathrm{O}_{2}$ could not trigger proteolytic cleavage of calcineurin (Figure 2). Consequently, DSCR1 may regulate other proteases as a component of a protease complex. It may function as an adaptor protein that facilitates a physical association between calcineurin and an unknown protease and provide structural stability to these complexes. Still, the detailed mechanisms remain to be discovered.

We also investigated the domain of DSCR1 that is involved in calcineurin proteolysis. As already reported, the C-terminal half of DSCR1 is required for calcineurin binding (Rothermel et al., 2000; Vega et al., 2002; Chan et al., 2005; Aubareda et al., 2006). In addition, overexpression of the C-terminal fragment leads to calcineurin cleavage (Figure 3). C-terminal region contains the FLISPPXSPP motif, which is conserved from yeast to human. Consistent with a previous study (Chan et al.,
2005), our results suggest that the FLISPPXSPP motif is not required for interaction with calcineurin, whereas the PXIIXT motif is needed for the interaction (Figure 3). As expected, truncation of the FLISPPXSPP motif had no effect on calcineurin cleavage. However, truncation of PXIIXT caused an unexpected result. Interestingly, when PXIIXT motif-deleted DSCR1 was overexpressed, calcineurin was cleaved at a different position. However, double deletion of both the FLISPPXSPP and PXIIXT motifs abrogated the ability of DSCR1 to accelerate the cleavage of calcineurin. Taken together, these results suggest that the PXIIXT motif alone is sufficient for calcineurin-DSCR1 interaction, but that the FLISPPXSPP motif also plays a role in recruitment and formation of a complex with an unknown protease, DSCR1 and calcineurin.

Finally, we confirmed these data under physiological conditions. We examined DSCR1 protein expression in skin fibroblasts from a DS patient. DSCR1 is overexpressed in the brain of DS fetuses (Fuentes et al., 2000). Here we showed that DSCR1 is overexpressed at the protein level in DS fibroblasts compared to normal fibroblasts. Likewise, DS fibroblasts exhibited accelerated cleavage of calcineurin compared to normal fibroblasts when treated with an equal amount of $\mathrm{H}_{2} \mathrm{O}_{2}$ (Figure 4). Moreover, knockdown of DSCR1 in DS fibroblasts completely blocked the protease activity induced by $\mathrm{H}_{2} \mathrm{O}_{2}$, suggesting that DSCR1 may be an essential component of a protease complex regulated by oxidative stress. 


\section{Methods}

\section{Cell culture}

To obtain primary cortical neuron cultures, embryos were surgically removed from ICR mice (E.15.5); their cortices were dissected and cultured as described (Koh et al., 1995). The skin fibroblast cells from a Down syndrome patient (D) and a normal person (N) were obtained from the Aging Cell Repository of the Coriell Cell Repository (CCR). Down syndrome fibroblast was AG08942 (21-yearold male patient) and control fibroblast was AG10803 (22-year-old healthy male). Fibroblast cells were grown in DMEM with $15 \%(\mathrm{v} / \mathrm{v})$ FBS and $50 \mathrm{ml}^{-1}$ streptomycin and penicillin. Human embryonic kidney (HEK) 293 cells and human neuroblastoma SK-N-SH cells were obtained from American Type Culture Collection and grown in DMEM containing $10 \%(\mathrm{v} / \mathrm{v}) \mathrm{FBS}, 50 \mathrm{U} / \mathrm{ml}$ streptomycin and penicillin.

\section{Transfection and DNA construct}

SK-N-SH cells were transfected using Lipofectamine reagent (Life Technologies, Carlsbad, CA) according to the manufacturer's protocol. HEK 293 cells were transfected by the calcium phosphate method. Expression vectors for DSCR1 mutants were constructed by subcloning PCR fragments into the pcDNA-FLAG vector (Clontech, Palo Alto, CA). Detailed information about the DNA constructs used in this study is available upon request.

\section{Adenoviral infection}

Recombinant adenoviruses were generated as previously described (He et al., 1998). DSCR1 cDNA containing a carboxy-terminal FLAG epitope tag was inserted into pAdTrack vector before recombination with pAdEasy-1 vector. Recombination between the pAdEasy-1 and pAdTrack vectors resulted in adenovirus green fluorescent protein (Ad-GFP). Viruses were propagated in the 293A cell line and prepared by freezing and thawing 4 times. Mouse primary cortical neurons were infected with adenoviruses and the infection efficiency was about $90-100 \%$. Cells were harvested for preparation of protein extracts 48 $\mathrm{h}$ after infection.

\section{SiRNA}

The siRNA nucleotides targeting human DSCR1 or ZAKI-4 and control siRNA targeting GFP were purchased from Dharmacon (Chicago, IL). The siRNA sequences were as follows: human DSCR1 siRNA, 5'-AAACGAGUCAGAAUAAACUUU-3'; human ZAKI-4 SiRNA, 5'-GUGACGUUCCAGCUAUUUAUU-3' and control GFP siRNA, 5'-GGCUACGUCCAGGAGCGCACCUU-3'. siRNA transfections were performed using Oligofectamine (Life Technologies) according to the manufacturer's instructions.

\section{RT-PCR}

Knockdown of specific genes was analyzed by RT-PCR. $\beta$-actin was used as a standard. The primer sequences were as follows: DSCR1 forward primer, 5'-GAGTTTCT-
GGGAAAGGAAAT-3' and reverse primer, 5'-AGCTTGGAGATGGCATATAA-3'; ZAKI-4, 5'-GCCAAACAGTTTCTCATCTC-3' and 5'-GCATGGAGCTCATACTTCTC-3'; $\beta$-actin, 5'-GGCATCCACGAAACTACCTT-3' and 5'-CTGTGTGGACTTGGGAGAGG-3.

\section{Immunoprecipitation and pull-down assay}

Immunoprecipitation and pull-down assays were described previously (Kim et al., 2008). Briefly, HEK293 cells were transfected with mammalian expression vectors for wild-type and truncated mutants of GST-tagged DSCR1 or Flagtagged DSCR1 in the presence of calcineurin expression plasmid. Lysates were prepared in lysis buffer (20 mM Tris, $150 \mathrm{mM} \mathrm{NaCl}, 0.5 \% \mathrm{NP}-40$, and $2 \mathrm{mM}$ PMSF, pH 7.4). Supernatants obtained by centrifugation at $12,000 \times g$ were incubated with glutathione-sepharose $4 \mathrm{~B}$ beads or protein-G beads pre-bound with anti-FLAG antibody for $2 \mathrm{~h}$ at $4^{\circ} \mathrm{C}$. The bead complexes were washed three times with lysis buffer; the proteins were then released in SDS sample buffer and detected by western blotting using the appropriate antibody.

\section{DSCR1 antibody}

We generated an anti-DSCR1 polyclonal antibody by immunizing rabbits with antigen peptide. The peptide sequence was KIIQTRRPEYTPIHLS, which is the proximal C-terminus of human DSCR1. We added a cysteine to the $\mathrm{N}$-terminus of this peptide and conjugated the peptide to keyhole limpet hemocyanin prior to immunization. AntiDSCR1 serum was affinity-purified by passage through a column of covalently linked DSCR1 immunization peptide.

\section{Acknowledgments}

This work was supported by KOSEF grants from the National Research Laboratory (ROA-2007-000-20002-0) and the Center for Functional Analysis for Human Genome (3344-20060070) to H.D.Y.

\section{References}

Aramburu J, Rao A, Klee CB. Calcineurin: from structure to function. Curr. Top. Cell. Regul. 2000; 36:237-95

Aubareda A, Mulero MC, Perez-Riba M. Functional characterization of the calcipressin 1 motif that suppresses calcineurin-mediated NFAT-dependent cytokine gene expression in human T cells. Cell Signal 2006;18:1430-8

Chan B, Greenan G, McKeon F, Ellenberger T. Identification of a peptide fragment of DSCR1 that competitively inhibits calcineurin activity in vitro and in vivo. Proc Natl Acad Sci USA 2005;102:13075-80

Crabtree GR. Calcium, calcineurin, and the control of transcription. J. Biol. Chem. 2001;276:2313-6

Crawford DR, Leahy KP, Abramova N, Lan L, Wang Y, Davies KJ. Hamster adapt78 mRNA is a Down syndrome critical region homologue that is inducible by oxidative stress. Arch 
Biochem Biophys 1997;342:6-12

Ermak G, Morgan TE, Davies KJ. Chronic overexpression of the calcineurin inhibitory gene DSCR1 (Adapt78) is associated with Alzheimer's disease. J Biol Chem 2001;276: 38787-94

Ermak G, Harris CD, Davies KJ. The DSCR1 (Adapt78) isoform 1 protein calcipressin 1 inhibits calcineurin and protects against acute calcium-mediated stress damage, including transient oxidative stress. FASEB J 2002;16: 814-24

Fuentes JJ, Genesca L, Kingsbury TJ, Cunningham KW, Perez-Riba M, Estivill X, de la Luna S. DSCR1, overexpressed in Down syndrome, is an inhibitor of calcineurinmediated signaling pathways. Hum Mol Genet 2000;9: 1681-90

Gong CX, Singh TJ, Grundke-lgbal I, Igbal K. Alzheimer's disease abnormally phosphorylated $\tau$ is dephosphoryulated by protein phosphatase-2B (Calcineurin). J. Neurochem. 1994;62:803-6

Harris CD, Ermak G, Davies KJ. Multiple roles of the DSCR1 (Adapt78 or RCAN1) gene and its protein product calcipressin 1 (or RCAN1) in disease. Cell Mol Life Sci 2005; 62:2477-86

He TC, Zhou S, da Costa LT, Yu J, Kinzler KW, Vogelstein B. A simplified system for generating recombinant adenoviruses. Proc Natl Acad Sci USA 1998;95:2509-14

Hilioti Z, Gallagher DA, Low-Nam ST, Ramaswamy P, Gajer $P$, Kingsbury TJ, Birchwood CJ, Levchenko A, Cunningham KW. GSK-3 kinases enhance calcineurin signaling by phosphorylation of RCNs. Genes Dev 2004;18:35-47

lizuka M, Abe M, Shiiba K, Sasaki I, Sato Y. Down syndrome candidate region 1, a downstream target of VEGF, participates in endothelial cell migration and angiogenesis. J Vasc Res 2004;41:334-44

Kashishian A, Howard M, Loh C, Gallatin WM, Hoekstra MF, Lai Y. AKAP79 inhibits calcineurin through a site distinct from the immunophilin-binding region. J Biol Chem 1998;273: 27412-9

Kim BY, Kim H, Cho EJ, Youn HD. Nur77 upregulates HIF- $\alpha$ by inhibiting pVHL-mediated degradation. Exp Mol Med 2008;40:71-83

Kingsbury TJ, Cunningham KW. A conserved family of calcineurin regulators. Genes Dev 2000;14:1595-604

Koh JY, Gwag BJ, Lobner D, Choi DW. Potentiated necrosis of cultured cortical neurons by neurotrophins. Science 1995;268:573-5

Lai MM, Burnett PE, Wolosker H, Blackshaw S, Snyder SH.
Cain, a novel physiologic protein inhibitor of calcineurin. J Biol Chem 1998;273:18325-31

Lange AW, Molkentin JD, Yutzey KE. DSCR1 gene expression is dependent on NFATc1 during cardiac valve formation and colocalizes with anomalous organ development in trisomy 16 mice. Dev Biol 2004;266:346-60

Lee JE, Kim H, Jang H, Cho EJ, Youn HD. Hydrogen peroxide triggers the proteolytic cleavage and the inactivation of calcineurin. J Neurochem 2007;100:1703-12

Lin X, Barber DL. A calcineurin homologous protein inhibits GTPase-stimulated Na-H exchange. Proc Natl Acad Sci USA 1996;93:12631-6

Michtalik HJ, Narayan AV, Bhatt N, Lin HY, Mulligan MT, Zhang SL, Crawford DR. Multiple oxidative stress-response members of the Adapt78 family. Free Radic Biol Med 2004;37:454-62

Rhee SG. Redox signaling: hydrogen peroxide as intracellular messenger. Exp Mol Med 1999;31:53-9

Rothermel B, Vega RB, Yang J, Wu H, Bassel-Duby R, Williams RS. A protein encoded within the Down syndrome critical region is enriched in striated muscles and inhibits calcineurin signaling. J Biol Chem 2000;275:8719-25

Seong JK, Kim DK, Choi KH, Oh SH, Kim KS, Lee SS, Um $\mathrm{HD}$. Proteomic analysis of the cellular proteins induced by adaptive concentrations of hydrogen peroxide in human U937 cells. Exp Mol Med 2002;34:374-8

Vandenbroucke K, Robbens S, Vandepoele K, Inzé D, Van de Peer Y, Van Breusegem F. Hydrogen peroxide-induced gene expression across kingdoms: A comparative analysis. Mol Biol Evol 2008; 25:507-16

Vega RB, Yang J, Rothermel BA, Bassel-Duby R, Williams RS. Multiple domains of MCIP1 contribute to inhibition of calcineurin activity. J Biol Chem 2002;277:30401-7

Vega RB, Rothermel BA, Weinheimer CJ, Kovacs A, Naseem $\mathrm{RH}$, Bassel-Duby R, Williams RS, Olson EN. Dual roles of modulatory calcineurin-interacting protein 1 in cardiac hypertrophy. Proc Natl Acad Sci USA 2003;100: 669-74

Yang J, Rothermel B, Vega RB, Frey N, McKinsey TA, Olson EN, Bassel-Duby R, Williams RS. Independent signals control expression of the calcineurin inhibitory proteins MCIP1 and MCIP2 in striated muscles. Circ Res 2000; 87:E61-E8

Yao YG, Duh EJ. VEGF selectively induces Down syndrome critical region 1 gene expression in endothelial cells: a mechanism for feedback regulation of angiogenesis? Biochem Biophys Res Commun 2004;321:648-56 